J. Clin. Chem. Clin. Biochem.

Vol. 15, 1977, pp. 503-508

\title{
Vollenzymatische Triglyceridbestimmung im Continuous Flow System am 12-Kanal-Analysator
}

\author{
Von H. M. Liebich, P. H. Müller, R. M. Schmülling, M. Eggstein \\ Medizinische Klinik, Abteilung Innere Medizin IV (Direktor: Professor Dr. M. Eggstein) der Universität Tübingen, \\ F. Stähler
}

Boehringer Mannheim GmbH, Biochemica Werk Tutzing

und $D$. Weißhaar

Medizinische Poliklinik Würzburg

(Eingegangen am 28. Dezember 1976/5. Mai 1977)

Herrn Prof. Dr. Dr. h. c. G. Schettler zum 60. Geburtstag gewidmet

Zusammenfassung: Eine vollenzymatische Triglyceridbestimmung mit Hilfe eines Lipase-Esterase-Gemisches zur Hydrolyse und nachfolgender enzymatischer Glycerinbestimmung wird in das Analysenspektrum eines continuous flow 12-Kanal-Analysators einbezogen.

Die Methode ist bis $7,9 \mathrm{mmol} / 1(700 \mathrm{mg} / 100 \mathrm{ml})$ linear. Die analytische Präzision liegt im Konzentrationsbereich von 1,5 bis $5,4 \mathrm{mmol} / 1$ ( $133 \mathrm{bis} 478 \mathrm{mg} / 100 \mathrm{ml}$ ) bei Variationskoeffizienten zwischen 0,8 und 4,8\%. Im unteren Meßbereich bei Konzentrationen um $0,7 \mathrm{mmol} / 1(62 \mathrm{mg} / 100 \mathrm{ml})$ ergibt sich unter Routinebedingungen bei 1140 Messungen ein mittlerer Variationskoeffizient von $7,2 \%$. Als mittlerer prozentualer Verschleppungskoeffizient wird für Triglycerid-Konzentrationen von 0,9 bis $7,7 \mathrm{mmol} / 1$ ( $80 \mathrm{bis} 680 \mathrm{mg} / 100 \mathrm{ml}) \mathrm{Q}=2,0$ ermittelt. Störende Einflüsse durch Bilirubin werden nicht beobachtet. Im Vergleich zur manuellen Methode nach Eggstein \& Kreutz ((1966) Klin. Wochenschr. 44, 262-267) liegen die Werte nach der vollenzymatischen Methode am 12-Kanal-Analysator um etwa $16 \%$ niedriger, korrigiert um einen additiven Faktor von $0,029 \mathrm{mmol} / 1(2,59 \mathrm{mg} / 100 \mathrm{ml})$. Bei den Richtigkeitskontrollen mit Kontrollseren wurde eine Differenz von $10 \%$ beobachtet.

\section{A fully enzymatic triglyceride determination in a continuous flow 12-channel analyzer}

Summary: A fully enzymatic triglyceride determination utilizing enzymatic hydrolysis with a lipase-esterase mixture and subsequent enżymatic glycerol determination, has been adapted for use in a continuous flow 12-channel-analyzer.

The method is linear up to $7.9 \mathrm{mmol} / \mathrm{l}(700 \mathrm{mg} / 100 \mathrm{ml})$. The analytical precision in the concentration range of 1.5 to $5.4 \mathrm{mmol} / \mathrm{l}$ ( 133 to $478 \mathrm{mg} / 100 \mathrm{ml}$ ) is characterized by relative standard deviations of 0.8 to $4.8 \%$. In the lower measuring range at concentrations around $0 . \overline{\mathrm{mmol}} / \mathrm{l}(62 \mathrm{mg} / 100 \mathrm{ml})$ a mean relative standard deviation of $7.2 \%$ is found for 1140 measurements under routine conditions. For triglyceride concentrations of 0.9 to $7.7 \mathrm{mmol} / 1$ ( 80 to $680 \mathrm{mg} / 100 \mathrm{ml}$ ) a mean relative coefficient of overspill $\mathrm{Q}=2.0$ is de termined. Bilirubin caused no observable interference in the determination. In comparișon with the manual method by Eggstein \& Kreutz ((1966) Klin. Wochenschr. $44,262-267)$ the results from the fully enżymatic method on the 12-channel-analyzer were lower by approximately $16 \%$, corrected by an additive factor of $0.029 \mathrm{mmol} / 1(2.59 \mathrm{mg} / 100 \mathrm{ml})$. The accuracy controls with control sera showed a difference of $10 \%$.

\section{Eịnleitung}

Die Einbeziehung der Triglyçeride neben dem Cholesterin in das Analysenspektrum eines Vielkanalanalysators erweitert das Screening-Profil dieses Gerätes um zwei für die Erkennung von Risikoindikatoren koronarer Herzkrankheiten wichtige Größen.

Seit Einführung der enzymatischen Triglyceridbestimmung über Glycerin nach alkalischer Verseifung durch Eggstein 
\& Kreutz (1) wurden mehrere Versuche mechanisierter Triglyceridbestimmungen beschrieben. Es handelt sich um fluorimetrische und kolorimetrische Verfahren, die halboder vollmechanisiert am Einkanal- oder Zweikanalanalysator durchgeführt wurden. Sie setzen eine Extraktion der Triglyceride mit Isopropanol oder Nonan-Isopropanol und die Adsorption störender Substanzen wie Phosphatide und Glucose aus dem Extrakt voraus und beruhen auf der Freisetzung des Glycerins durch alkalische Verseifung oder Umesterung, der Oxidation des Glycerins zu Formaldehyd und der Hantzsch-Reaktion zu 3,5-Diacetyl1,4-dihydrolutidin, das fluorimetrisch oder kolorimetrisch gemessen wird (2-7). Köhring \& Kattermann (7) bestimmten die Triglyceride zusammen mit Cholesterin halbmechanisiert am Zweikanalanalysator. Sämtliche Verfahren sind infolge der Extraktion methodisch aufwendig. Eine vollenzymatische Triglyceridbestimmung am Zentrifugalanalysator mit hydrolytischer Spaltung durch Lipase und enzymatischer Glycerinmessung wurde von Tiffany et al. (8) beschrieben.

Nach Einführung einer vollenzymatischen Triglyceridbestimmung, bei der die Hydrolyse der Triglyceride in Glycerin und Fettsäuren durch eine Lipase und eine Esterase katalysiert wird $(9,10)$, führten Kattermann et al. (11) die Bestimmung an einem Endwert-Automaten durch.

Diese Arbeit berichtet über die Adaption der vollenzymatischen Triglyceridbestimmung auf den 12-Kanal-Analysator SMA 12/60. Technische Durchführung, Präzision, Richtigkeit und Vergleichbarkeit der SMA 12/60-Ergebnisse mit denen der manuellen Methode (1) wurden untersucht.

\section{Methodik}

\section{Prinzip}

Die Methode beruht auf der Hydrolyse der Triglyceride mit Hilfe eines Lipase-Esterase-Gemisches (9) und nachfolgender enzymatischer Bestimmung des "Gesamtglycerins", das sich aus dem „freien Glycerin" und dem „Triglycerid-Glycerin“" zusammen- setzt (10). Die Triglyceride werden als unkorrigierte TriglyceridWerte angegeben, d. h. der Anteil des freien Glycerins wird nicht in Abzug gebracht.

\section{Geräte}

Die manuellen Triglyceridbestimmungen wurden durchgeführt am Photometer Eppendorf $1100 \mathrm{M}$

Wellenlänge: $334 \mathrm{~nm}$

Küvette: $1 \mathrm{~cm}$ Schichtdicke

Dem mechanisierten Verfahren lag folgende Gerätekonstellation zu Grunde:

Technicon Autoanalyzer SMA 12/60

Probenfrequenz 60 Proben $/ \mathrm{h}$

Probentisch IBM 1894

Permanent wirksame Probenidentifizierung über Kurzlochkarten Werteerfassung, Auswertung, Qualitätskontrolle und Statistik über Prozeßrechner IBM 1800, on-line Anschluß

Küvette: $2 \mathrm{~mm}$ Durchflußküvette

Wellenlänge: $340 \mathrm{~nm}$

Heizbadtemperatur: $37^{\circ} \mathrm{C}$

Dialysator: 12 Zoll

Fließschema s. Abbildung 1

Reagenzien und Durchführung für die manuelle Bestimmung nach Eggstein \& Kreutz:

Der Ansatz erfolgte mit den bei Bergmeyer (12) beschriebenen Reagenzien nach dem Originalverfahren.

für die vollenzymatische Bestimmung am SMA 12/60:

Die eingesetzten Enzyme und Substrate entsprachen den bei Wahlefeld ( 9 ) beschriebenen. Zusätzlich wurden im LipolyseReagenz Glucose-6-phosphat und Glucose-6-phosphatdehydrogenase verwendet, um das durch die im Primärkreis (Fließschema, Abb. 1) ablaufende Reaktion des Serum-Pyruvats oxidierte NADH wieder zu reduzieren. Auf diese Weise sollte ein NADH-Konzentrationssprung zwischen Primär- und Sekundärkreis verhindert werden.

Die Messungen wurden mit Testpackungen der Firma Boehringer Mannheim durchgeführt, Charge 7065403.

\section{Konzentrationen der Gebrauchslösungen}

Puffer-Substrat-Gemisch $0,05 \mathrm{mmol} / \mathrm{l}$ Triethanolamin-Puffer, $\mathrm{pH} 7,6$ $4 \mathrm{mmol} / 1$ Magnesiumchlorid $0,07 \mathrm{mmol} / \mathrm{l} \mathrm{NADH}$ $0,25 \mathrm{mmol} / \mathrm{l}$ ATP $0,2 \mathrm{mmol} / 1$ Phosphoenolpyruvat $0,5 \mathrm{~g} / 1$ Tween 20

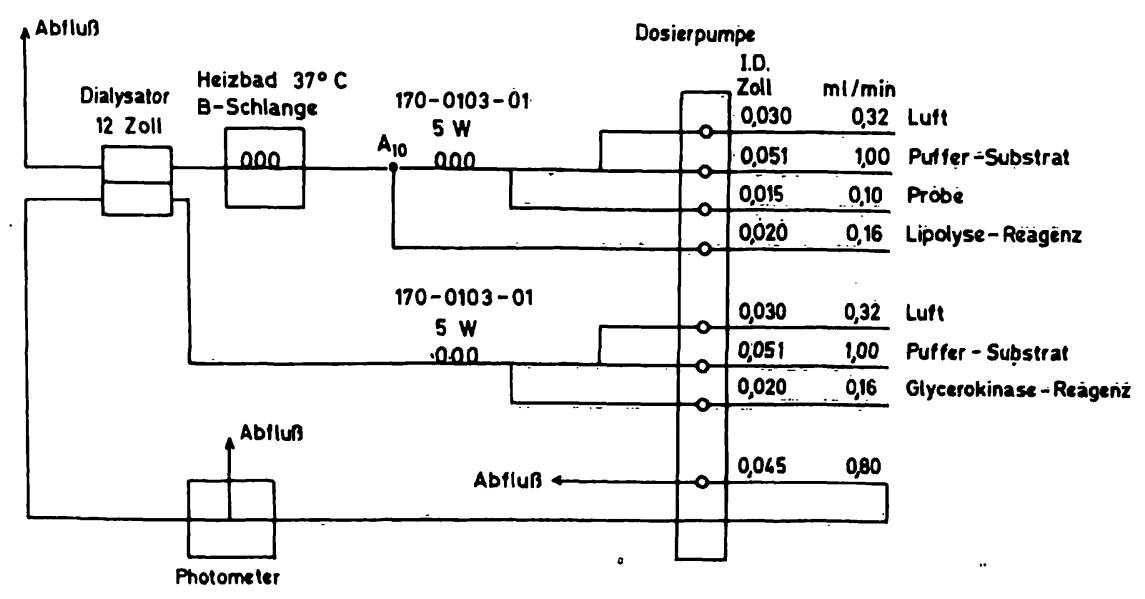

Abb. 1. Fließdiagramm für die vollenzymatische Triglyceridbestimmung am Auto-Analyzer SMA 12/60.. . 


$\begin{array}{ll}\text { Lipolyse-Reagenz } & 1,5 \mathrm{MU} / 1 \text { Lipase } \\ & 1,5 \mathrm{kU} / 1 \text { Esterase } \\ & 30 \mathrm{kU} / 1 \text { Lactatdehydrogenase } \\ & 5 \mathrm{kU} / 1 \text { Pyruvatkinase } \\ & 7,5 \mathrm{kU} / 1 \text { Glucose-6-phosphatdehydrogenase } \\ & 10 \mathrm{mmol} / 1 \text { Glucose-6-phosphat }\end{array}$

Glycerokinase-Reagenz 0,6 kU/1 Glycerokinase $30 \mathrm{kU} / 1$ Lactatdehydrogenase $5 \mathrm{kU} / 1$ Pyruvatkinase

Die technische Durchführung ist im Fließschema (Abb. 1) wiedergegeben. Die Verbindung zwischen Dialysator und Photometer ist so dimensioniert, daß eine Reaktionszeit von 1 Minute nicht unterschritten wird. Alle Lösungen wurden verschlossen im Kühlschrank bei $4^{\circ} \mathrm{C}$ aufbewahrt. Dabei sind die Gebrauchslösungen eine Woche haltbar.

\section{Kalibrierung}

Während der Erprobungsphase wurde ein stabilisierter wäßriger Primärstandard verwendet, der $20,8 \mathrm{mg}$ Glycerin in $100 \mathrm{ml}$ Lösung enthält, entsprechend $2,26 \mathrm{mmol} / 1$ Triglyceride bzw. $200 \mathrm{mg} / 100 \mathrm{ml}$ (Precimat Triglyceride $200 \mathrm{mg} / 100 \mathrm{ml}$, Boehringer Mannheim, Best. Nr. 15621).

Nach Einführung der vollenzymatischen Triglyceridbestimmung als Routineuntersuchung wurde die Kalibrierung mit dem für die übrigen Parameter des SMA 12/60 verwendeten SMA Reference Serum (Technicon Instruments Corporation, Charge BAJ 709) durchgeführt. Da für dieses Standardserum kein Sollwert für die Triglyceride angegeben ist, wurde laborintern in einer 100-fach Bestimmung am SMA $12 / 60$ der Sollwert mit $1,17 \mathrm{mmol} / 1$ $(104 \mathrm{mg} / 100 \mathrm{ml})$ ermittelt. Die Kalibrierung des Gerätes erfolgte mit dem angegebenen Primärstandard.

\section{Ergebnisse und Diskussion}

\section{Stabilität der Basislinie}

Die Überprüfung der Basislinie unter Verwendung der fertigen Gebrauchslösungen ergab in Tests über $45 \mathrm{~min}$ eine Schwankung von maximal einem Skalenteil nach oben oder unten, entsprechend $\pm 0,11 \mathrm{mmol} / 1$ Triglyceride $(10 \mathrm{mg} / 100 \mathrm{ml})$. Eine einseitige Drift war nicht festzustellen. Unter Routinebedingungen ergaben sich bei Serien von jeweils 20 Patientenproben, gefolgt von einer Präzisionskontrollprobe mit $0,7 \mathrm{mmol} / 1$ Triglycēride $(62 \mathrm{mg} / 100 \mathrm{ml})$, ebenfalls Schwankungen von maximal einem Skalenteil. Die Nachjustage der Basislinie nach jeweils 20 Proben wird empfohlen. Diese Frequenz stimmt mit dem von uns verwendeten Beschickungsschema des Probentellers für dạs SMA 12-Profil überein (13-15).

\section{Meßbereich und Linearität}

Für die manuelle vollenzymatische Triglyceridbestimmung wurde eine lineare Abhängigkeit des Meßsignals von der Triglycerid-Konzentration bis zu einer Konzentration von $7,9 \mathrm{mmol} / 1 \mathrm{l}(700 \mathrm{mg} / 100 \mathrm{ml})$ gefunden (10). Die Überprüfung des Linearitätsbereiches am 12-Kanal-Analysator erfolgte über eine Verdünnungsreihe. Ein Poolserum mit erhöhtem Triglycerid-Gehalt wurde in 10 Stufen mit physiol. NaCl-Lösung verdünnt und von jeder Konzentration eine 3-fach-Bestimmung durchgeführt $(9+1,8+2, \ldots, 1+9)$. Bei einem Korrelationskoeffizienten $r=0,999 \pm 0,004$ ergab sich auch am 12-Kanal-

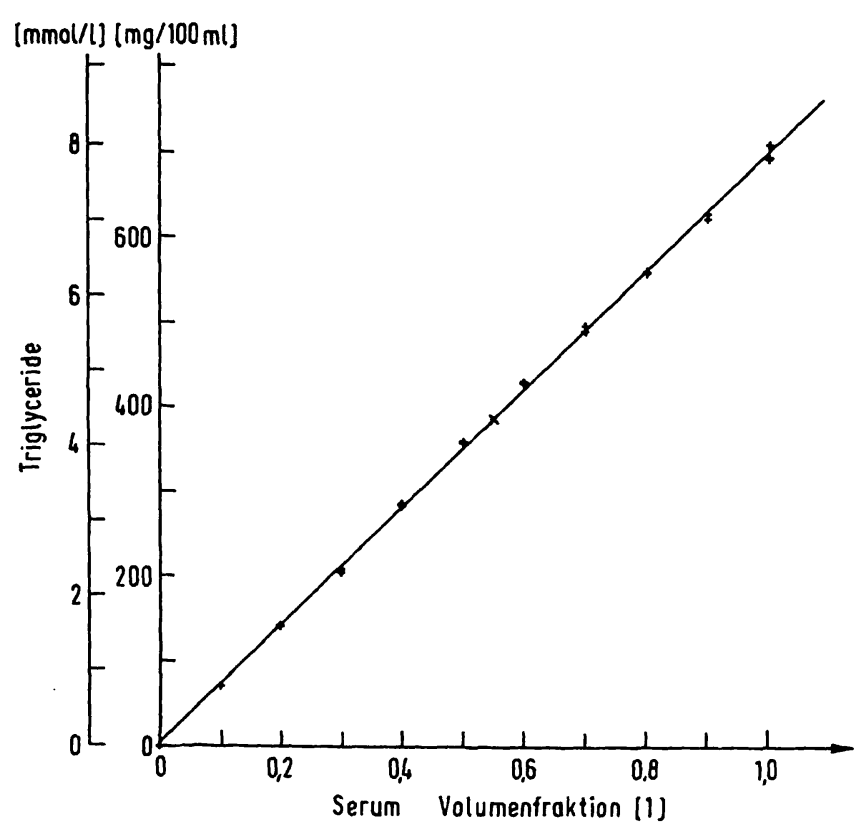

Abb. 2. Linearitätsprüfung der Triglyceridbestimmung. 3-fach Bestimmungen in der Verdünnungsreihe $(10+0$; $9+1 ; \ldots \ldots \ldots ; 2+8 ; 1+9)$ $y=69,68 x+0,06 \mathrm{mmol} / \mathrm{l} ; \mathrm{y}=69,68 \mathrm{x}+5,30 \mathrm{mg} / 100 \mathrm{ml}$ $r=0,999$

Analysator eine lineare Beziehung bis zu einer TriglyceridKonzentration von $7,9 \mathrm{mmol} / \mathrm{l}$ bzw. $700 \mathrm{mg} / 100 \mathrm{ml}$ (Abb. 2). Das Ende des linearen Bereiches liegt zwischen 7,9 und $10,2 \mathrm{mmol} / \mathrm{l}(700$ und $900 \mathrm{mg} / 100 \mathrm{ml})$. Unter Einhaltung einer Sicherheitsreserve wählen wir für Routinemessungen eine Begrenzung des Meßbereichs auf $6,9 \mathrm{mmol} / 1(602 \mathrm{mg} / 100 \mathrm{ml})$. Lipämische Seren werden mit physiol. NaCl-Lösung verdünnt.

\section{Verschleppung}

Die Überprüfung der im System auftretenden Verschleppung erfolgte über die Analyse von 3 Poolseren unterschiedlicher Konzentration in der Probensequenz $5 \mathrm{H}-$ $5 \mathrm{~N}-5 \mathrm{H}-5 \mathrm{M}-5 \mathrm{H}-5 \mathrm{~N}-5 \mathrm{M}-5 \mathrm{~N}[\mathrm{H}=$ hohe Konzentration $\bar{x}=7,7 \mathrm{mmol} / \mathrm{l}(680 \mathrm{mg} / 100 \mathrm{ml})$, $M=$ mittlere Konzentration $\bar{x}=3,1 \mathrm{mmol} / 1(274 \mathrm{mg} /$ $100 \mathrm{ml}), N=$ niedrige Konzentration $\bar{x}=0,9 \mathrm{mmol} / \mathrm{l}$ $(80 \mathrm{mg} / 100 \mathrm{ml})]$.

Als Ausdruck für die Verschleppung wird der Verschleppungskoeffizient $\mathrm{q}$ bzw. der prozentuale Verschleppungskoeffizient Q nach Hjelm (16) (auch bei Haeckel \& Porth (17)) gewählt

$$
q=\frac{\bar{B}-B_{1}}{B_{1}-\bar{A}} \quad Q=q \cdot 100
$$

$B_{1}=$ erster Wert einer Folgeserie B von 5 gleichen Proben

$\bar{A}=$ Mittelwert aus dem zweiten, dritten, vierten und fünften Wert einer Serie A.

$\bar{B}=$ Mittelwert aus dem zweiten, dritten, vierten und fünften Wert einer Folgeserie B. 
Tab. 1. Prozentuale Verschleppungskoeffizienten $H=$ hohe Konzentration $\overline{\mathrm{x}}=7,7 \mathrm{mmol} / 1 ; \mathrm{M}=$ mittlere Konzentration $\overline{\mathrm{x}}=3,1 \mathrm{mmol} / 1 ; \mathrm{N}=$ niedrige Konzentration $\overline{\mathrm{x}}=0,9 \mathrm{mmol} / \mathrm{l}$.

\begin{tabular}{ll}
\hline $\begin{array}{l}\text { Q 1 } \\
\text { (von der niedrigeren zur } \\
\text { höheren Konzentration) }\end{array}$ & $\begin{array}{l}\text { Q 2 } \\
\text { (von der höheren zur niedrigeren } \\
\text { Konzentration) }\end{array}$ \\
\hline $\mathrm{N} \rightarrow \mathrm{H} 2,86$ & $\mathrm{H} \rightarrow \mathrm{N} 2,13$ \\
$\mathrm{M} \rightarrow \mathrm{H}-0,24$ & $\mathrm{H} \rightarrow \mathrm{M} 1,47$ \\
$\mathrm{~N} \rightarrow \mathrm{M} 2,85$ & $\mathrm{H} \rightarrow \mathrm{N} 2,17$ \\
& $\mathrm{M} \rightarrow \mathrm{N} 2,33$ \\
\hline
\end{tabular}

Tab. 2. Präzision in der Serie

\begin{tabular}{llll}
\hline $\mathrm{N}=30$ & $\begin{array}{l}\text { Mittelwert } \overline{\mathrm{x}} \\
(\mathrm{mmol} / \mathrm{l})\end{array}$ & $\begin{array}{l}\text { Standard- } \\
\text { abweichung s } \\
\text { (mmol/l) }\end{array}$ & $\begin{array}{l}\text { Variations- } \\
\text { koeffizient VK } \\
(\%)\end{array}$ \\
\hline & & 0,75 & 2,2 \\
1,44 & 0,025 & 1,6 \\
1,92 & 0,059 & 3,1 \\
2,64 & 0,021 & 0,8 \\
5,42 & 0,040 & 0,8 \\
\hline
\end{tabular}

Für Verschleppungen von der niedrigen zur hohen Konzentration ergab sich als Mittel $Q_{1}=1,82$, für Verschleppungen von der hohen zur niedrigen Konzentration $Q_{2}=2,02$. Die Einzelwerte für $Q_{1}$ und $Q_{2}$ sind in Tabelle 1 wiedergegeben.

\section{Präzision der Methode}

Die „Präzision in der Serie“ wurde mit 5 Poolseren unterschiedlicher Konzentration geprüft, indem jeweils 30 Proben eines Konzentrationsbereiches hintereinander analysiert wurden. Dabei ergaben sich Variationskoeffizienten zwischen $0,8 \%$ und $3,1 \%$ (Tab. 2).

Für die „Präzision in der unterbrochenen Serie an mehreren Tagen" (Definition siehe 15) unter Routinebedingungen über einen Zeitraum von 28 Wochen $(n=1140)$ ergibt sich für den ungünstig niedrigen Konzentrationsbereich von $\bar{x}=0,71 \mathrm{mmol} / 1(62,8 \mathrm{mg} / 100 \mathrm{ml})$ eine Standardabweichung $s=0,051 \mathrm{mmol} / \mathrm{l}(4,5 \mathrm{mg} / 100 \mathrm{ml})$ und ein Variationskoeffizient $\mathrm{VK}=7,2 \%$. In Abbildung 3 ist der Rechnerausdruck für die Präzisionskontrolle (Kontrollkarte) wiedergegeben.

In einer zusätzlichen Untersuchung ermittelten wir die „Präzision in der unterbrochenen Serie an einem Tag“" und die „Präzision von Tag zu Tag“ nach einem Randomisierungsverfahren. An 5 aufeinanderfolgenden Tagen wurden in 5 Poolserumproben unterschiedlicher Triglyceridkonzentrationen $\overline{\mathrm{x}}=0,79 \mathrm{mmol} / \mathrm{l}(70 \mathrm{mg} / 100 \mathrm{ml})$ bis $\bar{x}=5,41 \mathrm{mmol} / \mathrm{l}(479 \mathrm{mg} / 100 \mathrm{ml}) 5$-fach-Bestimmungen durchgeführt. Die Proben wurden während der Untersuchungszeit portioniert bei $4^{\circ} \mathrm{C}$ aufbewahrt und täglich aufs neue zufallsverteilt, d. h. die 5 Proben einer Konzentration wurden nicht hintereinander analysiert.

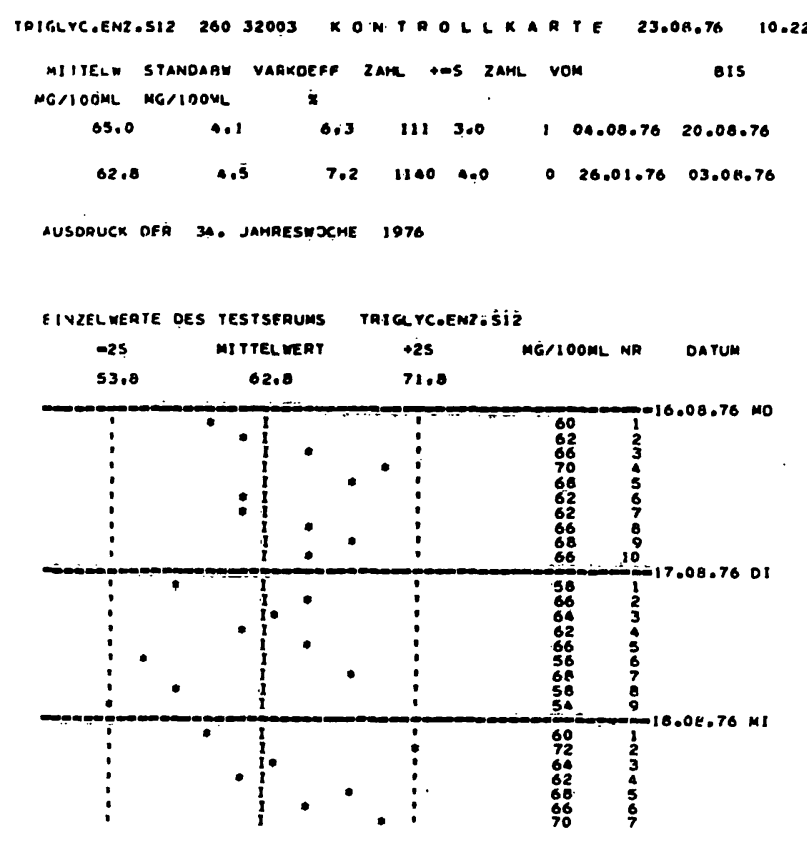

Abb. 3. Rechnerausdruck zur Präzisionskontrolle in der ,unterbrochenen Serie an mehreren Tagen" (berechnet für 2 und 28 Wochen, Ausschnitt aus Kontrollkarte über 3 Tage).

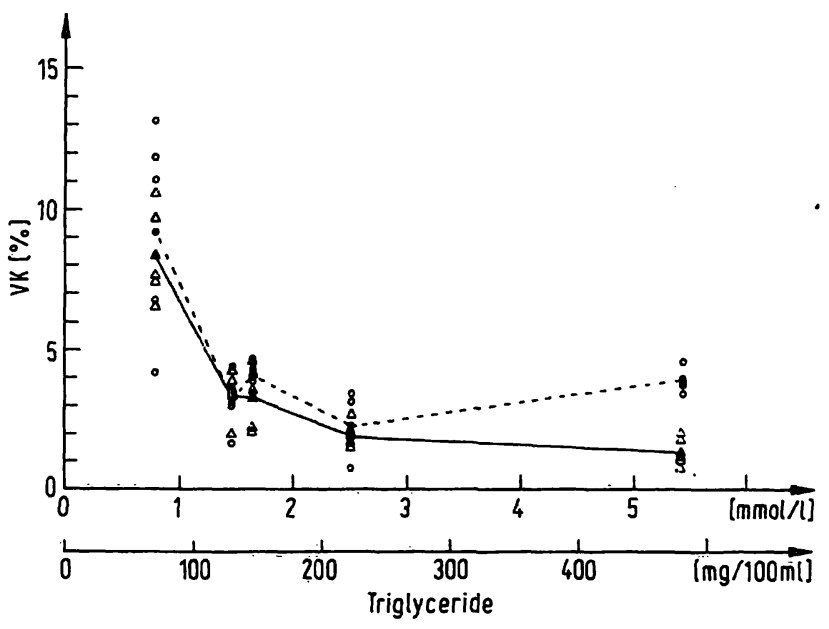

Abb. 4. Einzel-Variationskoeffizienten $(\Delta, c)$ und mittlere Variationskoeffizienten $(\Delta, \bullet)$ in Abhängigkeit von der Triglyceridd-Konzentration. "Präzision in der unterbrochenen Serie an einem Tag" $(\Delta)$ und "Präzision von Tag zu Tag" ( 0 ) bei Zufallsverteilung.

$\triangle$ _ـ ,Präzision in der unterbrochenen Serie an einem Tag" $(n=5)$

$\bullet-\cdot \bullet$ „Präzision von Tag zu Tag“ $(n=5$ )

Nach diesem Verfahren läßt sich aus einer kleinen Analysenzahl eine Aussage über die analytische Präzision machen unter Einschluß von Verschleppungsfehlern. Aus der Versuchsanordnung ergeben sich für jeden der 5 Konzentrationsbereiche 5 Angaben über die „Präzision in der unterbrochenen Serie an einem Tag" $(n=5)$ und aus den jeweils ersten, zweiten, dritten, vierten und fünften Bestimmungen an jedem Tag für jeden Konzentrationsbereich 5 Angaben über die „Präzision von Tag zu Tag"' $(n=5)$. Das Verfahren wurde ebenfalls. für die Präzision bei der manuellen vollenzymatischen Tñglyceridbestimmung angewandt und beschrieben (10). 
Die am SMA 12/60 erzielten Ergebnisse sind in Abbildung 4 wiedergegeben. Mit Ausnahme des niedrigsten Konzentrationsbereiches lagen die Variationskoeffizienten für beide Arten der Präzision zwischen 0,8 und 4,8\%. Es wird daraus deutlich, daß die analytische Präzision für die Triglyceridbestimmung im Routinebetrieb ausreichend gut und von Tag zu Tag-Schwankungen weitgehend unabhängig ist. Bei niedrigen Konzentrationen um $0,79 \mathrm{mmol} / 1(70 \mathrm{mg} / 100 \mathrm{ml})$ streuen die Variationskoeffizienten stärker und liegen zwischen 3,4-13,2\%.

\section{Störungen bei Hyperbilirubinämie}

Der Einfluß von Bilirubin wurde durch Zumischen einer Bilirubin-Verdünnungsreihe untersucht. Bei BilirubinKonzentrationen bis $265 \mu \mathrm{mol} / 1(15,5 \mathrm{mg} / 100 \mathrm{ml})$ wurde keine Störung beobachtet.

\section{Rich tigkeitsk on trolle}

Die Ergebnisse der an Kontrollseren überprüften Richtigkeit der Methode sind in Tabelle 3 zusammengefaßt. Dabei wurden die SMA 12/60-Werte den nach der manuellen Methode (1) ermittelten Werten gegenübergestellt. Den Vergleichen lastet an, daß nicht jeder KontrollserumHersteller Angaben über die Berücksichtigung des freien Glycerins macht. Unter Beachtung dieses Unsicherheitsfaktors ergeben sich gegenüber der manuellen Methode am SMA 12/60 im Mittel um 10\% niedrigere Werte. Dies entspricht den Ergebnissen der Richtigkeitskontrollen und des Methodenvergleichs zwischen manueller vollenzymatischer Bestimmung und manueller Bestimmung nach alkalischer Verseifung (10).

Tab. 3. Richtigkeitskontrolle mit Kontrollseren

\begin{tabular}{llll}
\hline $\begin{array}{l}\text { Kontrollserum } \\
\text { (Charge) }\end{array}$ & $\begin{array}{l}\text { angegebener } \\
\text { Sollwert, } \\
\text { Methode nach } \\
\text { Eggstein/Kreutz } \\
\text { (mmol/1) }\end{array}$ & SMA 12/60 & $\begin{array}{l}\text { Abwei- } \\
\text { chung vom } \\
\text { Sollwert }\end{array}$ \\
\hline & 1,31 & 1,17 & -10 \\
\hline $\begin{array}{l}\text { Validate N (031634) } \\
(\mathrm{N}=8)\end{array}$ & 0,64 & -8 \\
$\begin{array}{l}\text { Validate A (0552054) } \\
(\mathrm{N}=8)\end{array}$ & 0,70 & 2,58 & -2 \\
$\begin{array}{l}\text { Liponorm (51) } \\
(\mathrm{N}=2)\end{array}$ & $2,63^{1}$ & 3,20 & -8 \\
$\begin{array}{l}\text { Leder-Trol } \\
(2907-301)(\mathrm{N}=5)\end{array}$ & 3,49 & 0,96 & -17 \\
$\begin{array}{l}\text { Monitrol II (38 AB) } \\
(\mathrm{N}=7)\end{array}$ & $1,16^{2}$ & 1,34 & -19 \\
$\begin{array}{l}\text { Kontrollogen L (412) } \\
(\mathrm{N}=2)\end{array}$ & $1,65^{2}$ & 1,25 & -9 \\
$\begin{array}{l}\text { Precilip (430 AB) } \\
(\mathrm{N}=2)\end{array}$ & $1,38^{1}$ & 1,18 & -8 \\
$\begin{array}{l}\text { Precilip (442 AB) } \\
(\mathrm{N}=14)\end{array}$ & $1,29^{1}$ & & \\
\hline
\end{tabular}

1 Rückkorrektur auf unkorrigierte Triglyceride nach den Angaben des Herstellers

2 keine Angaben über Korrektur

\section{Methodenvergleich an Patientenproben}

Der Vergleich zwischen vollenzymatischer Triglyceridbestimmung am SMA 12/60 und der Originalmethode nach Eggstein \& Kreutz erfolgte an einem Kollektiv von 76 Seren von Patienten mit normalen und erhöhten Triglyceridspiegeln (Konzentrationsbereich 0,75-6,9 mmol/1 bzw. $66-613 \mathrm{mg} / 100 \mathrm{ml}$ ) und erstreckte sich über 12 Tage. Die Kalibrierung des SMA 12/60 erfolgte

a) mit dem SMA Reference Serum, in dem der Sollwert nach Kalibrierung mit einem primären GlycerinStandard am SMA 12/60 bestimmt wurde und

b) mit einem Patientenserum, dessen Triglyceridgehalt nach der manuellen Referenzmethode (1) durch eine 5-fach Bestimmung ermittelt wurde.

Die Abbildungen 5 und 6 zeigen das Ergebnis der Korrelationsanalysen. Als Regressionsgleichung $y=b x+a$ ergab sich, wenn der SMA 12/60 nach a) kalibriert wurde (vgl. Abb. 5):

$$
y=0,843 x+0,029 \mathrm{mmol} / 1 ; \quad r=0,993 \pm 0,012
$$

bzw. $y=0,843 x+2,59 \mathrm{mg} / 100 \mathrm{ml} ; \quad r=0,993 \pm 0,012$

Der Korrelationskoeffizient $\mathrm{r}=0,993$ zeigt, daß die beiden Methoden vergleichbar sind. Die SMA 12-Werte liegen aber um $16 \%$ tiefer, korrigiert um einen additiven Faktor von $0,029 \mathrm{mmol} / 1(2,59 \mathrm{mg} / 100 \mathrm{ml})$. Damit kommt zu der für die manuelle vollenzymatische Triglyceridbestimmung bekannten Differenz von $10 \%$ (10)

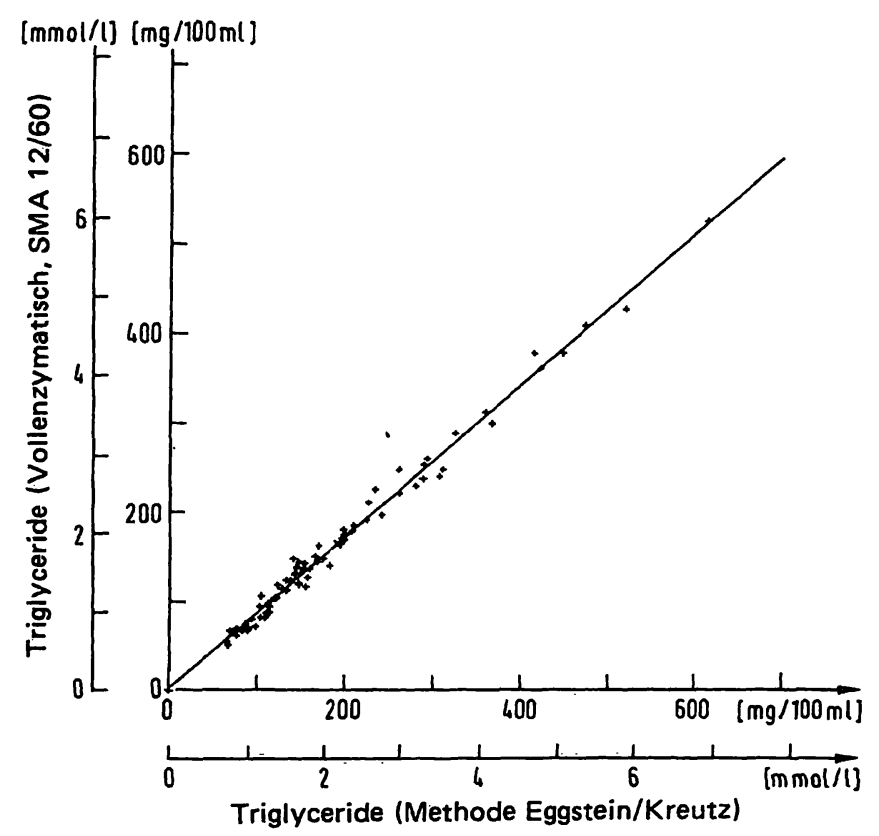

Abb. 5. Korrelation der Methoden $\mathrm{x}=$ Methode nach Eggstein \& Kreutz (1) $y=$ vollenzymatische Methode am SMA 12/60

Kalibrierung mit SMA Reference Serum, in dem der Sollwert über einen primären Glycerin-Standard am SMA $12 / 60$ bestimmt wurde.

$\mathrm{n}=76$

$y=b x+a$

$\mathrm{b}=0,843 \pm 0,010$

$\mathrm{a}=0,029 \mathrm{mmol} / \mathrm{l}=2,59 \mathrm{mg} / 100 \mathrm{ml}$

$r=0,993 \pm 0,012$ 


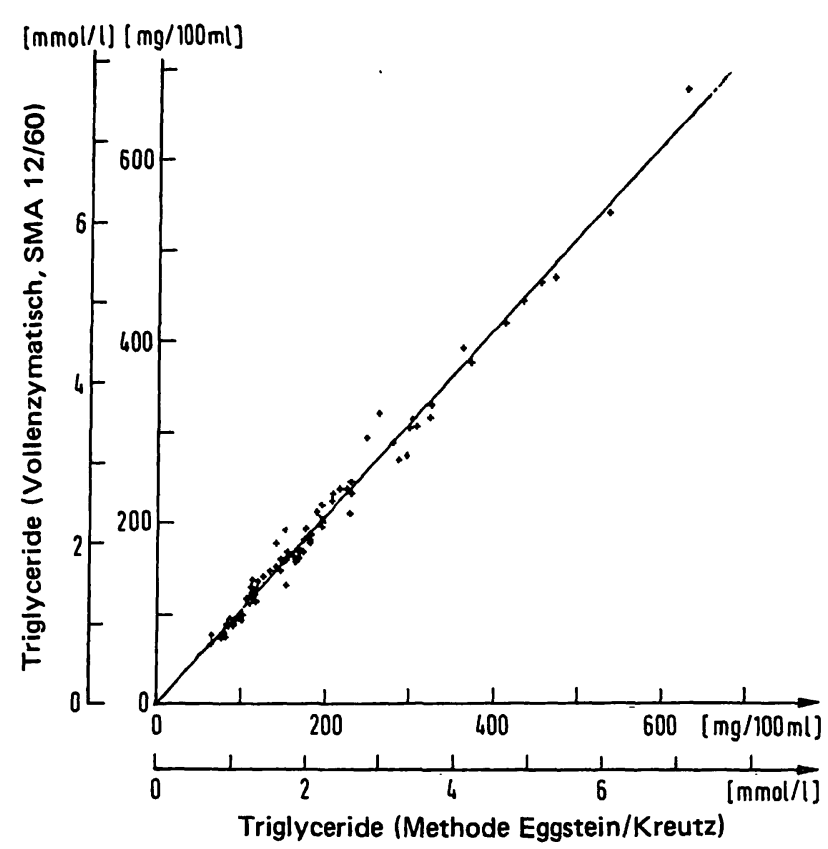

Abb. 6. Korrelation der Methoden $\mathrm{x}=$ Methode nach Eggstein \& Kreutz (1) $y=$ vollenzymatische Methode am SMA 12/60 Kalibrierung mit Patientenserum, in dem der Sollwert nach Eggstein \& Kreutz bestimmt wurde. $\mathrm{n}=76$

$\mathrm{y}=\mathrm{bx}+\mathrm{a}$

$\mathrm{b}=1,027 \pm 0,014$

$\mathrm{a}=0,001 \mathrm{mmol} / \mathrm{l}=0,103 \mathrm{mg} / 100 \mathrm{ml}$

$r=0,992 \pm 0,013$

eine weitere Differenz von etwa $6 \%$ hinzu. Legt man die manuelle Methode (1) als Referenzmethode zugrunde und führt die Kalibrierung des SMA 12/60 nach b), also mit einem Patientenserum durch, so ergibt sich für den Methodenvergleich mit denselben 76 Patientenseren die in Abbildung 6 dargestellte Korrelationsanalyse mit der Regressionsgleichung $y=b x+a$

$$
y=1,027 x+0,001 \mathrm{mmol} / 1 ; \quad r=0,992 \pm 0,013
$$

bzw. $y=1,027 x+0,103 \mathrm{mg} / 100 \mathrm{ml} ; \mathrm{r}=0,992 \pm 0,013$

Bei diesem Vorgehen wird eine sehr gute Korrelation zwischen dem manuellen und dem continuous-flow Verfahren erreicht. Die Werte sind praktisch identisch $(b=1,027 \pm 0,014 ; a=0,001 \mathrm{mmol} / \mathrm{l})$.

\section{Praktikabilität}

Die vollenzymatische Triglyceridbestimmung läßt sich am SMA 12/60 mit einer theoretischen Probenfrequenz von 60 Proben/h durchführen. Bei dem von uns gewählten Beschickungsschema entspricht dies 40 Proben/h (14). Um aussagekräftige Verlaufskontrollen zu bekommen, muß darauf geachtet werden, daß die Proben für die SMA 12/60 Üntersuchung nach fettfreier Abendmahlzeit etwa $12 \mathrm{~h}$ später nüchtern entnommenes Blut enthalten. Nachteile der an das continuous flow-Verfahren adaptierten vollenzymatischen Triglyceridbestimmung sind

1. die bis jetżt noch relativ hohen Kosten, die sich bei 200 Patientenseren pro Tag unter Berücksichtigung des Reagenzienverbrauchs während der Kalibrierphasen des Gerätes auf einen Richtwert von $0,80 \mathrm{DM}$ pro Bestimmung belaufen,

2. die bisher nicht durchgeführte Korrektur um den Anteil an freiem Glycerin und

3. die unbefriedigende Kalibrierung mit einem wäßrigen Glycerinstandard.

\section{Danksagung}

Wir danken dem technischen Assistenten Hern A. Sashegyi für seine gute und exakte Mitarbeit.

\section{Literatur}

1. Eggstein, M. \& Kreutz, F. H. (1966), Klin. Wochenschr. 44, 262-267.

2. Kessler, G. \& Lederer, H. (1966), In Automation in Analytical Chemistry, Technicon Symposia 1965 (Skeggs, Jr., L. T., ed.) Mediad, New York, S. 341.

3. Royer, M. E. \& Ko, H. (1969), Anal. Biochem. 29, 405-416.

4. Noble, R. P. \& Campbell, F. M. (1970), Clin. Chem. 16, 166-170.

5. Mc Lellan, G. H. (1971), Clin. Chem. 17, 535-537.

6. Björksten, F. (1972), Clin. Chim. Acta 40, 143-152.

7. Köhring, B. \& Kattermann, R. (1974), Z. Klin. Chem. Klin. Biochem. 12, 282-286.

8. Tiffany, T. O., Morton, J. M., Hall, E. M. \& Garrett, Jr., A. S. (1974), Clin. Chem. 20, 476-481.

9. Wahlefeld, A. W., (1974), Triglyceride: Bestimmung nach enzymatischer.Verseifung, in: Methoden der enzymatischen Analyse (Bergmeyer, H. U., ed.), 3. Auflage, S. 1878-1882, Verlag Chemie, Weinheim.

10. Müller, P. H., Schmülling, R.-M., Liebich, H. M., Eggstein, M., Stähler, F. \& Stinshoff, K. (1977), J. Clin. Chem. Clin. Bịochem. 15, 457-464.

11. Kattermann, R., Köhring, B. \& Bunne, B. (1976), Dtsch. Med. Wochenschr. 101, 933-957.

12. Eggstein, M. \& Kuhlmann, E., (1974), Triglyceride und Glycerin, in: Methoden der enzymatischen Analyse (Bergmeyer, H. U., ed.), 3. Auflage, S. 1871-1878, Verlag Chemie, Weinheim.

13. Schmülling, R.-M. (1970), Analysenspektrum und Labororganisation, Technicon Symposium 70, Bad Homburg v. d. H.

14. Schily, K., Eggstein, M., Knodel, W., Allner, R., Henig, S. \& Kuhlmann, E. (1969), Z. Klin. Chem. Klin. Biochem. 7, 66-71.

15. Schmülling, R.-M., Liebich, H. M., Locher, M., Mildner, I. \& Eggstein, M. (1973), Z. Klin. Chem. Klin. Biochem. 11, $513-520$.

16. Hjelm, M. (1968), Z. Analyt. Chemie 243, 781-790.

17. Haeckel, R. \& Porth, A. J. (1972), Z. Klin. Chem. Klin. Biochem. 10, 91-94.
Prof. Dr. med. Manfred Eggstein, Dr. rer. nat. Hartmut Liebich Medizinische Universitätsklinik Abteilung Innere Medizin IV Otfried-Müller-Straße 10 D-7400 Tübingen 